\title{
Effects of inclination angle on a shock-accelerated heavy gas column
}

\author{
D. Olmstead, C. R. Truman, P. Wayne \& P. Vorobieff \\ Department of Mechanical Engineering, The University of New Mexico, \\ USA
}

\begin{abstract}
When a shock encounters a multiphase interface at an oblique angle, threedimensional (3D) flow effects are produced. Experiments using advanced optical diagnostics seek to elucidate the 3D nature of the flow as it transitions to turbulence. Planar laser-induced fluorescence (PLIF) images capture the development of flow instabilities in a shock-accelerated heavy gas column. Early time images show the counter-rotating vortex pair (CRVP) associated with the Richtmyer-Meshkov instability (RMI) both for normal planar and for oblique shocks, with the cores of the vortex pair parallel to the axis of the original gas column. For the oblique case, a shear-driven Kelvin-Helmholtz instability (KHI) also develops along the axis of the column due to $3 \mathrm{D}$ vorticity deposition. The influence of inclination angle of the column with respect to the shock direction on this secondary instability and thus upon the fully 3D flow, is assessed. The 3D data collected in these experiments is essential to the validation of numerical codes predicting a range of problems from scramjets to supernovae.
\end{abstract}

\section{Introduction}

Understanding the evolution of instabilities caused by accelerating a medium of nonuniform density (single-phase or multiphase) is of critical interest for many challenging technological frontiers. For the well-characterized Rayleigh-Taylor instability [1,2], perturbations on the interface between the fluids of two different densities are amplified by sustained acceleration only when the higher density fluid is accelerated toward the lighter density fluid [3]. However, when the acceleration is impulsive rather than sustained, (e.g., its source is a shock wave rather than a 
gravity field), perturbations on the interface are always unstable, and the resulting Richtmyer-Meshkov instability (RMI) [4,5] causes rapid mixing and transition to turbulence in the region of the interface.

An important motivation for RMI studies because is the dramatically accelerated mixing at the interface. This mixing can be utilized for accelerated fuel/air mixing in combustion [6], or can have a detrimental effect as in reducing inertial confinement fusion yields by mixing fuel with impurities [7]. Understanding of RMI has also helped correlate models and observations for supernovæ [8], as well as improve hydrocode validation [9].

In the case of a circular column of heavy gas in a lighter gas accelerated by a shock wave, RMI initially manifests as a pair of counter-rotating vortex columns (CRVP) when viewed in the cross-section of the initial column [10-13]. This is due to the baroclinic vorticity deposition on each side of the column, due to misalignment between the pressure and density gradients. The former is planar (shock front), the latter is circular (cross-section of the column). As the result, two large-scale counter-rotating vortices form in the cross-sectional plane.

Similarly, baroclinic vorticity can be deposited in the plane of the column axis, provided that the column is inclined relative to the shock front, so that the gradients of density and pressure are misaligned at all points on the density interface. This vorticity deposition is not unlike that on an inclined planar interface [14], and in effect produces a shear layer along the density interface. Subsequently the samesign vorticity does not coalesce into a single large vortex. Instead, any smallwavelength perturbation becomes amplified through Kelvin-Helmholtz instability, eventually contributing to development of fully $3 \mathrm{D}$ disordered flow and transition to turbulence.

\section{Experimental setup}

The University of New Mexico Inclinable Shock Tube is depicted in Fig. 1a. Mach 1.13 to Mach 2.0 shock waves are produced by pressurizing the driver section with helium. When a precisely controlled prescribed pressure is reached, the polyester diaphragm separating the driver section from the driven section is ruptured, releasing the shock into the driven section. The latter is initially filled with air at ambient pressure. The speed of the shock wave is verified with two high speed pressure transducers at both ends of the driven section. From the driven section, the shock travels into the test section that has three aluminum walls and one acrylic wall.

A $6.25 \mathrm{~mm}$ diameter circular laminar jet of sulfur hexafluoride $\left(\mathrm{SF}_{6}\right)$ mixed with $1 \%$ acetone vapor (tracer) by volume is injected vertically down through the test section. The jet is stabilized by an annular co-flow of air as shown in Fig. 1b, to minimize perturbations in the initial conditions. Oblique shock interaction with the vertical jet is achieved by pivoting the entire shock tube arrangement to the desired inclination, as shown in Fig. 1c. In this paper, inclinations of $0^{\circ}$ and $20^{\circ}$ are presented. 


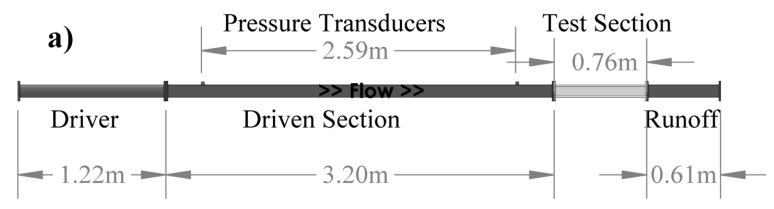

b)

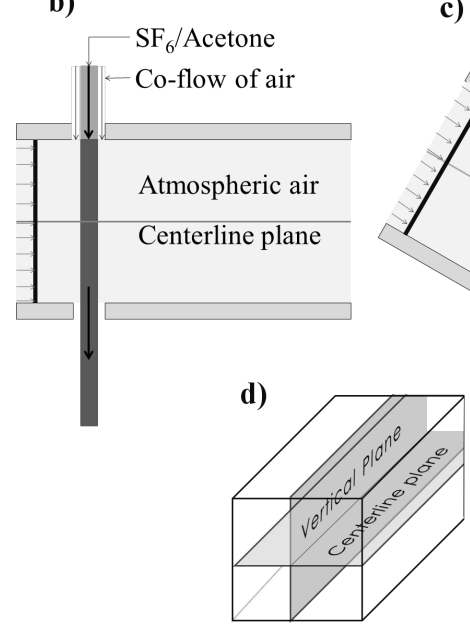

c)

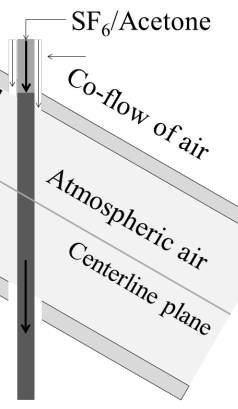

Figure 1: Drawing of UNM inclinable shock tube (a). Sketch of the normal shock (b) and $20^{\circ}$ inclined shock (c) initial condition column and laser sheets. The heavy gas column is injected vertically and initially stabilized by gravity. To produce an oblique angle between the plane of the shock front and the axis of the column, the entire shock tube is inclined with respect to horizontal. The laser sheets are perpendicular and pass through the midpoint of the tunnel as seen in the sketch (d). Individual images in the two planes are acquired in separate experimental runs with highly repeatable conditions.

A planar cross-section of the test section is illuminated with a frequency-quadrupled Nd-YAG pulsed laser emitting at $266 \mathrm{~nm}$ for about $5 \mathrm{~ns}$. The UV pulse causes the acetone mixed with the $\mathrm{SF}_{6}$ to fluoresce at $480 \mathrm{~nm}$. The UV sheet is oriented to be parallel to the shock direction and to pass through the centerline of the test section, so that it is oriented either vertically (vertical plane) or parallel to the floor of the test section (centerline plane), as shown in Fig. 1d. Images are acquired with a four-megapixel, 16-bit cooled CCD digital camera which captures a single fluorescence event in a $40 \times 40 \mathrm{~mm}$ field of view each time the shock tube is fired. Mach numbers vary by less than $0.5 \%$ from the prescribed value, and images from as many as 40 shots with the same nominal Mach number are used to reveal evolution of instabilities over time. 
To enable comparison of data at multiple Mach numbers and density ratios, a non-dimensional time parameter described in Eq. 1 is utilized. This time parameter is derived from the linear growth theory of RMI [15]. For the case of a circular cylinder, the wavenumber $k=2 \pi / d$ where $d$ is the diameter of the cylinder. The Atwood number $A=\left(\rho_{2}-\rho_{1}\right) /\left(\rho_{2}+\rho_{1}\right)$, where $\rho_{2}$ and $\rho_{1}$ are the heavy and light gas densities, respectively. For $\mathrm{SF}_{6}$ and air, $A=0.67$. For a traveling shock wave as in a shock tube, $\Delta V$ is the velocity of the air piston behind the shock wave. Data presented is all taken between the arrival of the incident shock wave and the time when either the expansion fan reflected from the upstream end of the driver or the shock reflected off the downstream wall of the shock tube reach the test section. It can be safely assumed that this piston velocity is constant during this time. The final term in Eq. 1 is the time since shock arrival at the center of the heavy gas column at $t_{0}$.

$$
\tau=k A \Delta V\left(t-t_{0}\right)
$$

\section{Results and discussion}

The evolution of the instability shows several distinct phases with interactions between the instabilities created due to the column inclination and the instabilities created due to the circular cross-section of the column.

When accelerated by a Mach 1.7 normal shock, the shock wave is lensed as it passes through the heavy gas column, as shown in Fig. 2, column a, $\tau=2$. This lensing (or shock focusing) is due to the speed of sound in $\mathrm{SF}_{6}$ being appreciably slower than in air. It creates the spike visible in the middle of the image at $\tau=8$ while the baroclinic vorticity deposition around the remainder of the column rolls up into two counter-rotating vortex columns seen at $\tau=20$. These vortices develop secondary instabilities at $\tau=25$ and then begin to mix aggressively, suggesting transition to turbulence has begun at $\tau=30$. The evolution of the same column with a Mach $1.7,20^{\circ}$ oblique shock is shown in Fig. 2, column b. The shock passage observed at $\tau=2$ is very similar to that observed in the normal shock case, as is the spike and early vortex formation seen at $\tau=8$ and $\tau=16$. The secondary instabilities that form are distinctly different as small wavelength, small amplitude instabilities are present along the outside edges of the vortices at $\tau=18$. These occur at a much earlier time than that seen for normal shock acceleration. The morphology of the final mixed column seen at $\tau=25$ is very similar to the normal shock accelerated column, however, mixing occurs at a much earlier time, as it could be expected from the earlier onset and greater prominence of secondary instabilities. A similar evolution pattern is observed for the Mach 2.0 shock wave, as shown in Fig. $3 a$ and b.

The vertical plane through the center of the column for the planar normal shock acceleration shows that the upstream edge develops random amplitude and spacing breakups and diamond patterns appear where the counter-rotating vortices are developing secondary instabilities as seen in Fig. 3c and finally transitioning to turbulent flow throughout. For oblique shock acceleration, the column appears thinner as for the normal shock due to compression and advection in to the CRVP, 


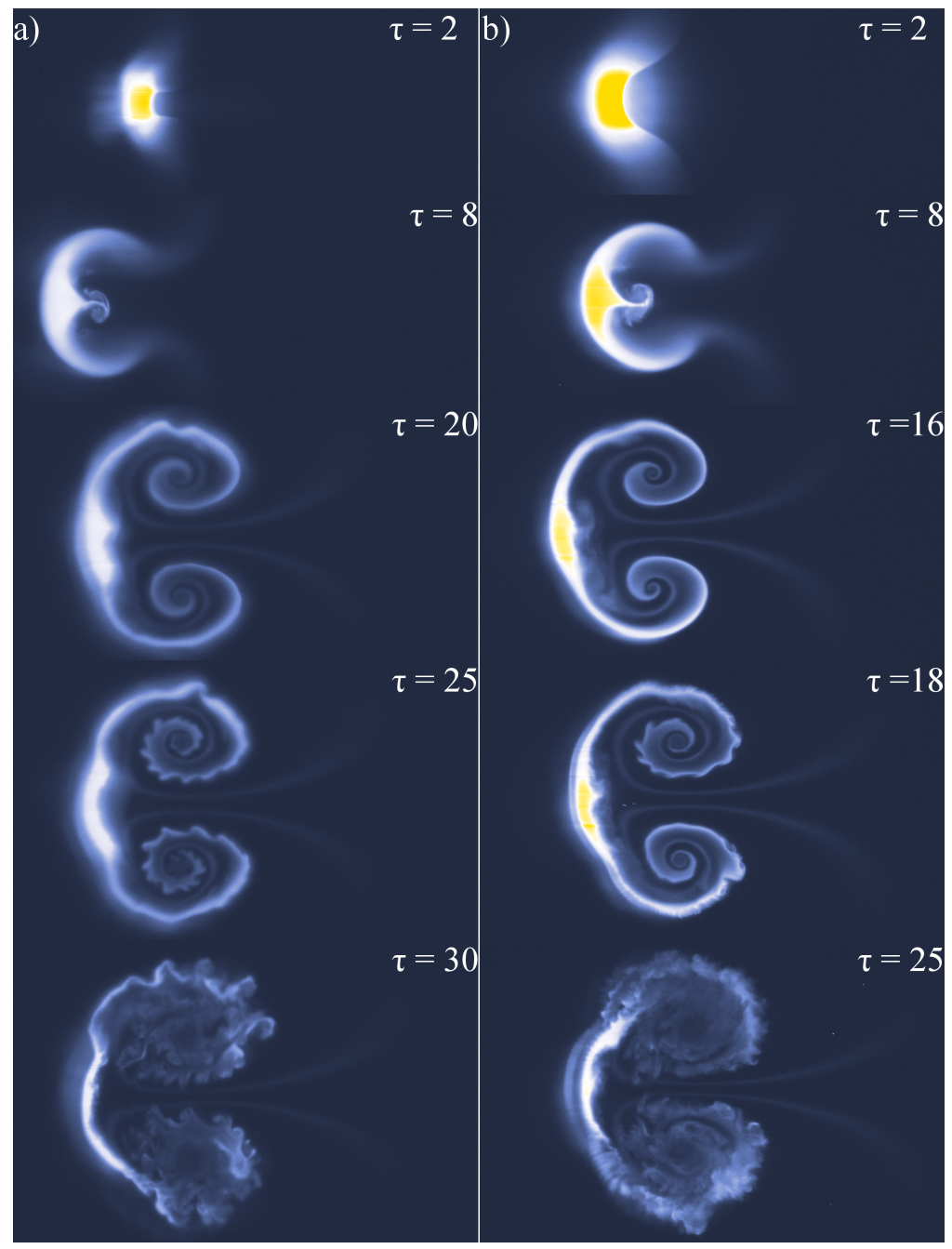

Figure 2: The growth of the RM instability on a circular cylinder accelerated by a Mach 1.7 normal shock is viewed using PLIF in the centerline plane in column (a). It is characterized by shock focusing with spike formation, initial counter-rotating vortex pair roll-up, onset of secondary instabilities in the vortices, and finally transition to turbulent flow. The evolution when accelerated by a $20^{\circ}$ oblique Mach 1.7 shock wave is shown in column (b). The principal change is the appearance of very small, pervasive secondary instabilities at an earlier time (b: $\tau=18$ ) than for normal shock acceleration (a: $\tau=25$ ). The secondary instabilities seen for normal shock accelerated flow still appear, however they break up more quickly. 
but very similar sized small wavelength co-rotating waves appear pointed down the column as shown on the left in Fig. 4. These waves quickly merge and cause a rapid transition to turbulence in the flow as seen in Fig. 3d.

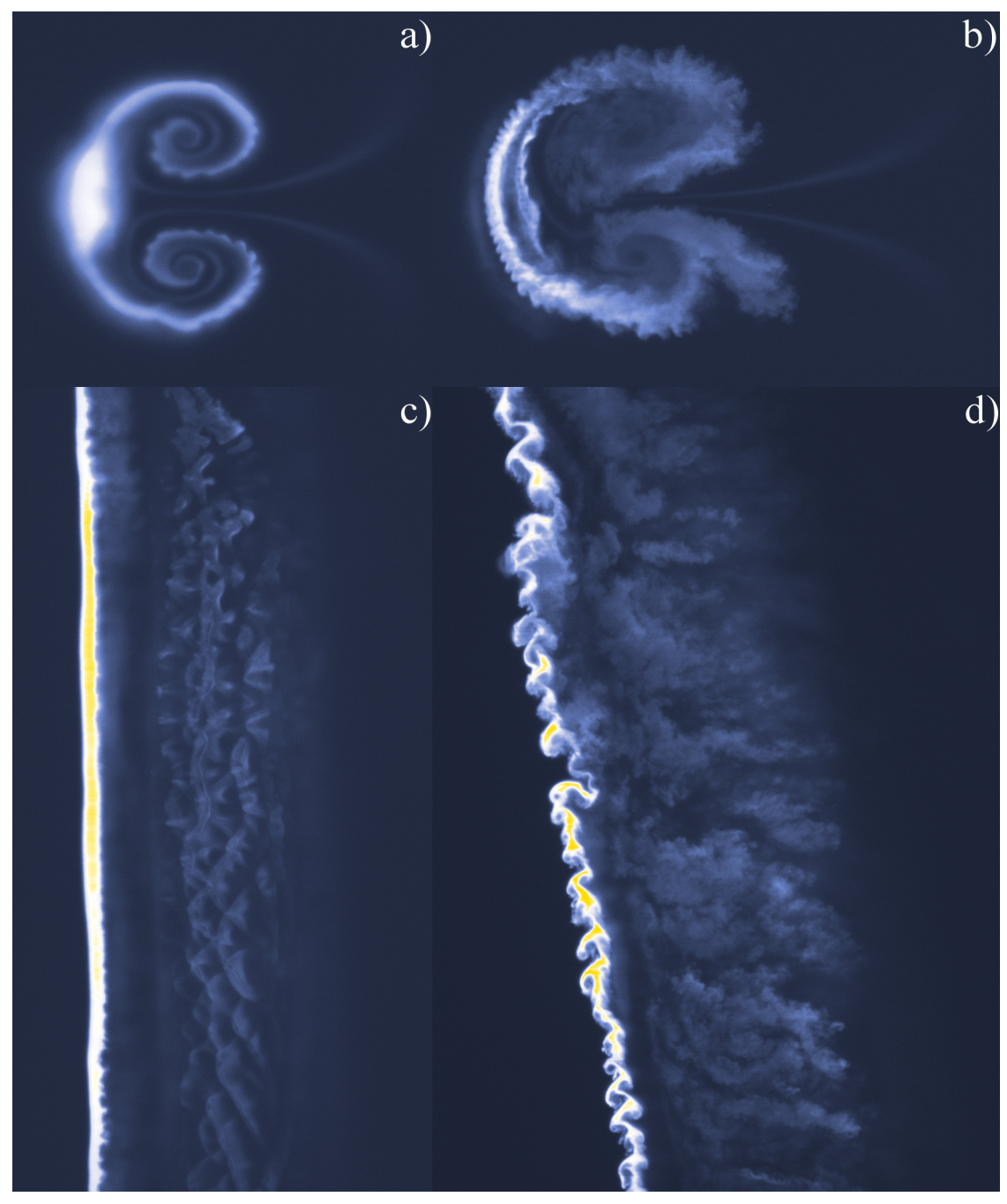

Figure 3: Centerline cross-sections ( $\mathrm{a}$ and $\mathrm{b}$ ) and corresponding vertical mid-plane images (c and d) for a Mach 2.0 incident shock wave. The shock wave is normal ( $\mathrm{a}$ and $\mathrm{c}$ ) or at a $20^{\circ}$ angle ( $\mathrm{b}$ and d) to the column axis, and both are at $\tau=25$. Secondary instabilities begin to appear in the normal shock wave images, but are significantly evolved everywhere except the upstream (left) edge of both images ( $b$ and d). 


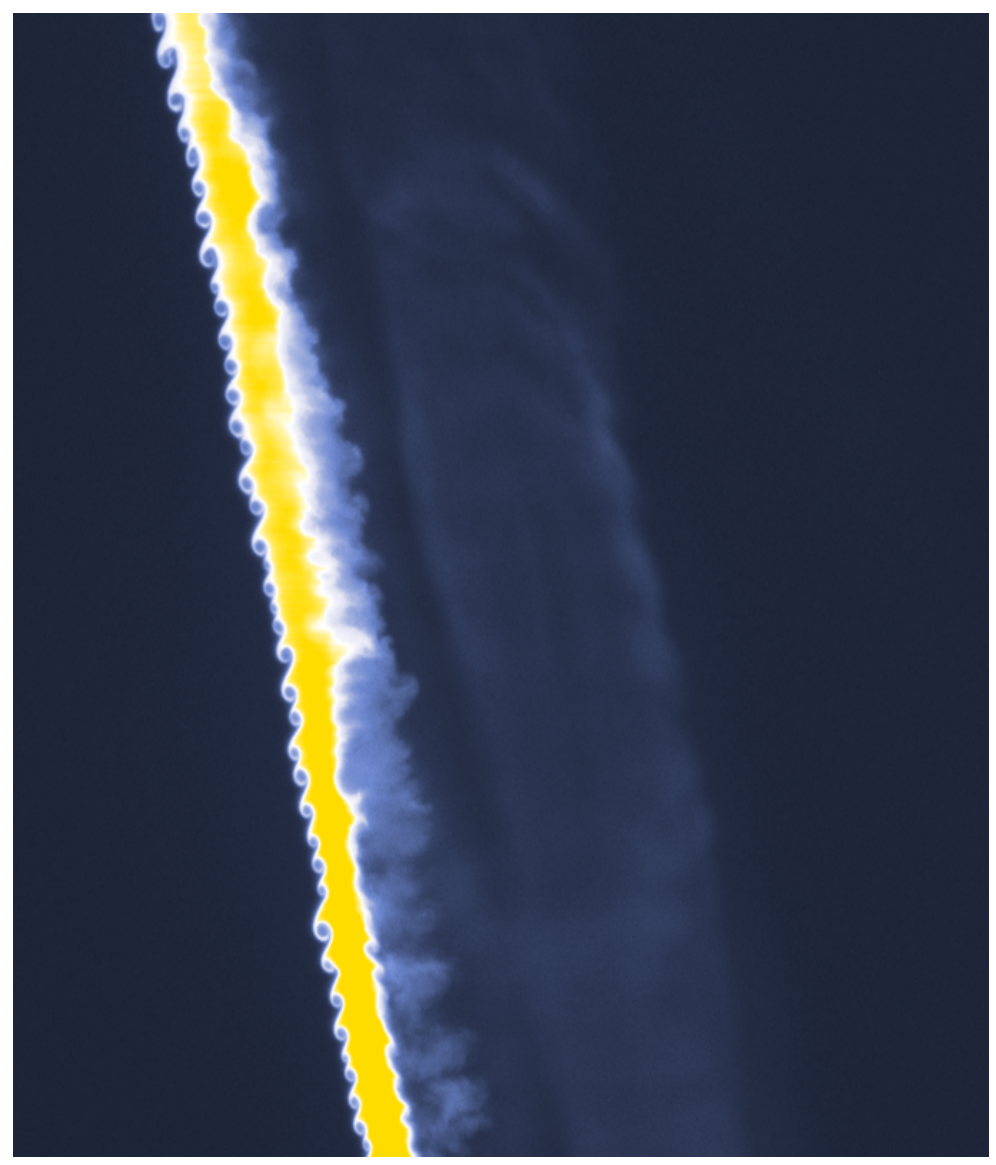

Figure 4: Vertical plane image of a Mach 2.0, $20^{\circ}$ oblique shock wave at $\tau=14$ showing vertical waves forming on the upstream (left) edge of the column. The waves are all of similar size and are all pointing down the column.

To quantify the change in the evolution of the instabilities in the centerline plane, the streamwise extent of the mixing zone was measured. A Matlab code was written to locate the relevant feature in the image (large-scale CRVP) and to determine its bounding rectangle based on dynamic thresholding over lines of consecutive pixels. The code was run for two different thresholds, as early and latetime images have different morphology and statistics. The resulting measurements in Fig. 5 show that, despite the apparent differences in the secondary instability onset and type for normal and oblique shock accelerated columns, with proper time scaling, there is no appreciable difference in the streamwise mixing-zone extent with Mach number or inclination. Because of this, different metrics for quantifying mixing and transition must be examined, including histogram analysis to detect 
changes in the fluorescence intensity distribution due to mixing, a combined streamwise and spanwise feature measurement to capture the rapid growth of features due to formation of secondary instabilities.

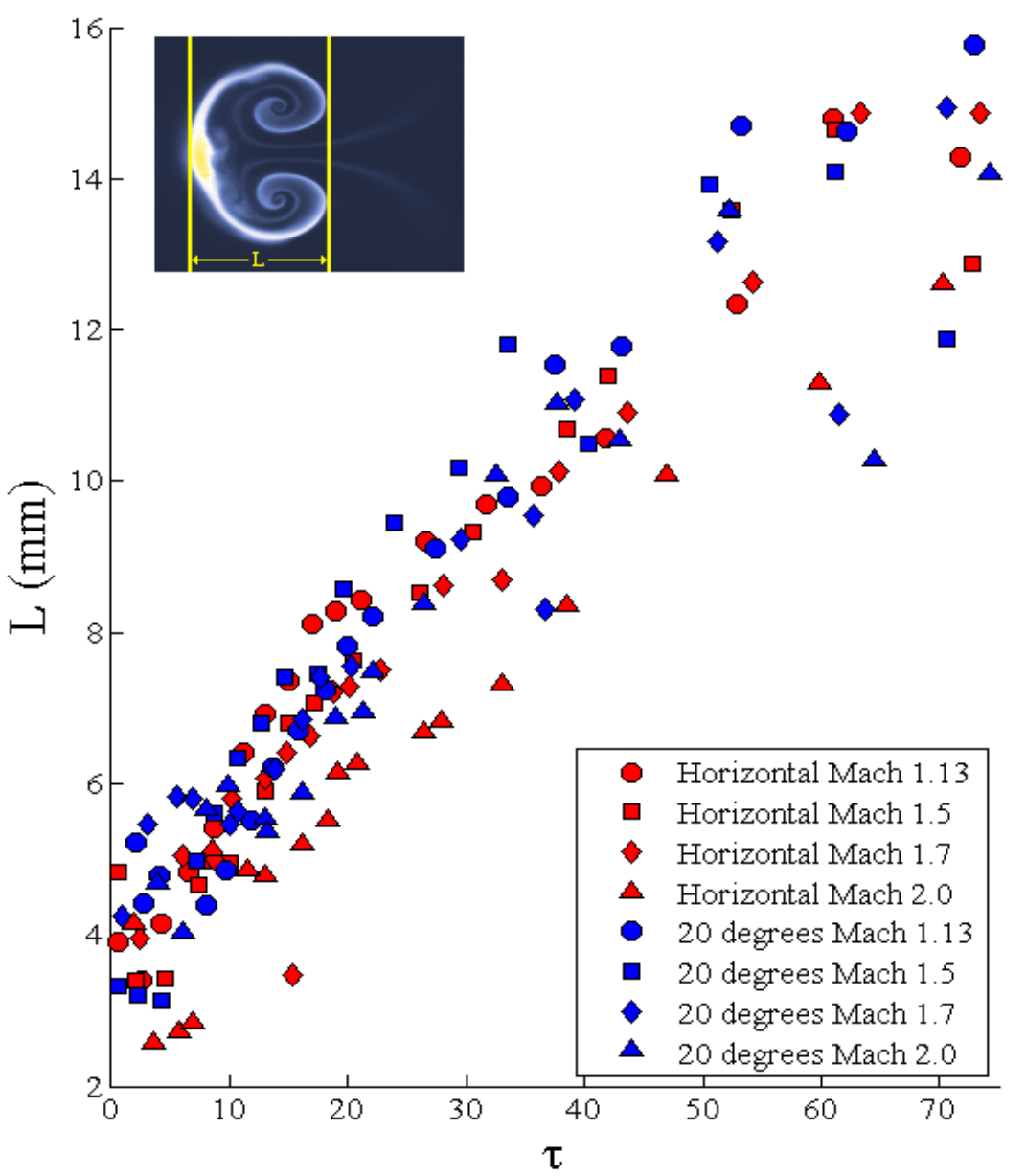

Figure 5: Streamwise extent of the mixing zone $L$ in $\mathrm{mm}$, measured using the centerline cross-section images for Mach numbers 1.13 to 2.0 at inclinations of $0^{\circ}$ (normal shock, red) and $20^{\circ}$ (oblique shock, blue). With time scaling $\tau$, growth curves for different Mach numbers appear to collapse. There is no discernible difference between $0^{\circ}$ and $20^{\circ}$ cases. 


\section{Conclusion}

Oblique shock acceleration of a circular column of heavy gas shows distinctly different instability growth patterns from those observed for planar normal shock acceleration. Despite changes in the type and rate of growth of the secondary instabilities, the overall streamwise extent of the large-scale counter-rotating vortices remains largely unchanged. This indicates that while smaller, earlier, secondary instabilities can change the time to turbulent transition, they do not change the overall ability of the large coherent vortices to feed energy to smaller vortices and thus complete the turbulent energy cascade more rapidly. Future work will focus on measuring velocity fields simultaneously with density fields to assist with turbulent transition quantification.

\section{Acknowledgement}

This research is supported by the US National Nuclear Security Administration (NNSA) grant DE-NA-0002220.

\section{References}

[1] Lord Rayleigh (J. W. Strutt). Investigation of the character of the equilibrium of an incompressible heavy fluid of variable density. Proceedings of the London Mathematical Society, 14:170-177, 1883.

[2] G. I. Taylor. The instability of liquid surfaces when accelerated in a direction perpendicular to their planes. Proceedings of the Royal Society of London. Series A, Mathematical and Physical Sciences, 201(1065):192-196, 1950.

[3] D.J. Lewis. The instability of liquid surfaces when accelerated in a direction perpendicular to their planes. II. Proceedings of the Royal Society of London. Series A, Mathematical and Physical Sciences, 202(1068):81-96, 1950.

[4] Robert D. Richtmyer. Taylor instability in shock acceleration of compressible fluids. Technical Report LA-1914, Scientific Laboratory of the University of California, Los Alamos, New Mexico, July 1954.

[5] E. E. Meshkov. Instability of the interface of two gases accelerated by a shockwave. Izv. AN SSSR, Mekh. Zhidk. Gaza, 4(5):151-157, 1969.

[6] F. E. Marble, E. E. Zukoski, J. W. Jacobs, G. J. Hendricks, and I. A. Waitz. Shock enhancement and control of hypersonic mixing and combustion. Technical Report AIAA 90-1981, 1990.

[7] John D. Lindl, Robert L. McCrory, and E. Michael Campbell. Progress toward ignition and burn propagation in inertial confinement fusion. Physics Today, 45(9):32-40, 1992.

[8] D Arnett. The role of mixing in astrophysics. Astrophysical Journal Supplement Series, 127(2):213-217, April 2000.

[9] Brian Fishbine. Code validation experiments. Los Alamos Research Quarterly, pages 6-14, 2002. 
[10] J. W. Jacobs. The dynamics of shock accelerated light and heavy gas cylinders. Physics of Fluids A, 5(9):2239-2247, 1993.

[11] C Tomkins, K Prestridge, P Rightley, P Vorobieff, and R Benjamin. Flow morphologies of two shock-accelerated unstable gas cylinders. Journal of Visualization, 5(3):273-283, 2002.

[12] P. Vorobieff, N.-G. Mohamed, C. Tomkins, C. Goodenough, M. MarrLyon, and R. F. Benjamin. Scaling evolution in shock-induced transition to turbulence. Physical Review E, 68(6):065301, December 2003.

[13] S. Kumar, G. Orlicz, C. Tomkins, C. Goodenough, K. Prestridge, P. Vorobieff, and R. Benjamin. Stretching of material lines in shockaccelerated gaseous flows. Physics of Fluids (1994-present), 17(8):082107, August 2005.

[14] Jacob A. McFarland, Jeffrey A. Greenough, and Devesh Ranjan. Simulations and analysis of the reshocked inclined interface Richtmyer-Meshkov instability for linear and nonlinear interface perturbations. Journal of Fluids Engineering, 136(7):071203 1-11, May 2014.

[15] Michael J. Anderson. Oblique shock interactions with perturbed density interfaces. PhD Dissertation, Mechanical Engineering, University of New Mexico, 2011. 